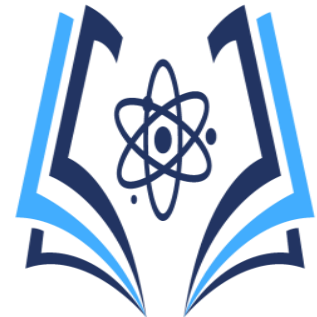

Patrones culturales y su influencia en la sexualidad de

\title{
los adolescentes
}

\section{Cultural patterns and their influence on adolescent sexuality \\ Patrones culturales y su influencia en la sexualidad}

\author{
Mercy Juleydi Rojas Villavicencio. Lic. Enf. ${ }^{1}$ \\ Virginia Esmeralda Pincay Pin. Mg. Gs. ${ }^{2}$ \\ María Elena Pincay Cañarte Mg. Gs. ${ }^{3}$ \\ Gina Rosa Alonso Muñiz $\mathrm{Mg} \mathrm{Em}^{4}$
}

${ }^{1}$ Universidad Estatal del Sur de Manabí, mercy.rojas@unesum.edu.ec, https://orcid.org/0000-0003-1999-938X

${ }^{2}$ Universidad Estatal del Sur de Manabí, virginia.pincay@unesum.edu.ec, https://orcid.org/0000-0001-8776-543

${ }^{3}$ Universidad Estatal del Sur de Manabí, maria.pincay@unesum.edu.ec. https://orcid.org/0000-0003-4110-9275

${ }^{4}$ Universidad Estatal del Sur de Manabí, Gina.alonzo@unesum.edu.ec , https://orcid.org/0000-0001-8776-5433

Correo de contacto: mercy.rojas@unesum.edu.ec

Aprobado: 18-06-2021

\section{Resumen}

La adolescencia periodo de transición entre la niñez y adultez donde ocurren cambios físicos, fisiológicos, sociales, emocionales y madurez sexual que promueve un desarrollo saludable, el objetivo de la investigación es determinar los patrones culturales que influyen en la sexualidad de las y los adolescentes de la comunidad de Chade del cantón Jipijapa provincia de Manabí. La población objeto de estudio fueron 30 adolescentes hombres y mujeres, la metodología aplicada fue descriptiva y analítica, se aplicó encuestas dando como resultado que los patrones culturales son creencias, mitos, costumbres, tradiciones y prácticas de la población, manifestaron además que la principal causa de embarazo en las adolescentes es el desconocimiento de la sexualidad, la mitad de ellos expresaron que sus padres si les educan, mientras el restante, no reciben educación sexual en sus hogares, otros que la educación sobre la sexualidad la recibieron en la unidad educativa, se concluye que una parte de los adolescentes si tienen conocimiento sobre los patrones culturales y la influencia en la sexualidad, sin embargo, existe un porcentaje significativo que no conoce mucho del tema, como: prevenir embarazos no deseados y enfermedades de transmisión sexual utilizando métodos anticonceptivos. Durante el estudio se encontró un caso de una adolescente de 17 años que es madre, vive con su pareja en un hogar estable. Para fortalecer la enseñanza y mejorar los patrones culturales de los y las adolescentes se proyectó una propuesta educativa.

Palabras claves: Cultura sexual, embarazos no deseados, conocimiento sexual.

\begin{abstract}
Adolescence is the transition period between childhood and adulthood where physical, physiological, social, emotional changes and sexual maturity occur that promote healthy development, the objective of the research is to determine the cultural patterns that influence the sexuality of adolescents from the Chade community of the Jipijapa canton, Manabí province. The population under study were 30 male and female adolescents, the applied methodology was descriptive, analytical, a survey was applied resulting in that the cultural patterns are beliefs, myths, customs, traditions and practices of the population, it was also stated that the main cause of pregnancy in adolescents is ignorance of sexuality, half of them expressed that their parents do educate them, while the rest do not receive sexual education in their homes, others that education about sexuality was received in the educational unit, It is concluded that a part of adolescents do have knowledge about cultural patterns and the influence on sexuality, however, there is a significant percentage that do not know much about the subject, such as: preventing unwanted pregnancies and sexually transmitted diseases using contraceptive methods . During the study, a case of a 17-year-old adolescent who is a mother, lives with her partner in a stable home, was found. To strengthen teaching and improve the cultural patterns of adolescents, an educational proposal was projected.
\end{abstract}

Keywords: Sexual culture, unwanted pregnancy, sexual knowledge.

Introducción 
La adolescencia es el período comprendido entre los 10 y 19 años, es una etapa de profundo cambios psicológicos y sociales. El individuo progresa desde la aparición inicial de las características sexuales secundarias hasta la madurez sexual. En esta etapa es importante una adecuada educación tanto en la prevención de embarazos a temprana edad e infecciones de transmisión sexual, favoreciendo de esta forma la adquisición de conductas protectoras en el ejercicio de sus sexualidades. La pubertad y el desarrollo sexual son etapas de cambios constantes, donde el deseo y los impulsos sexuales dominan a una parte racional que no está del todo desarrollada (1).

Liliana Cervantes, en su investigación año 2018, factores que influyen en el desarrollo de la sexualidad de los adolescentes en la ciudad de Santa Martha, concluyo que la gran problemática en la sexualidad de los jóvenes adolescentes es la causa masiva de la influencia de las redes sociales, donde han provocado un mal concepto de la sexualidad llevándolo de un componente del ser humano privado e individual a un aspecto público y morboso que ha contribuido que experimenten e interpreten su sexualidad completamente mal entendida (2).

Karin Zuberbi Calle Callo, expresó que los factores que influyen en el inicio de las relaciones sexuales en los adolescentes son el factor familia, individual, sociocultural y educativo (3). Yoannie Cuñat London de Guevara, en su investigación refleja que existe un incremento de inadecuados comportamientos y aptitudes de los adolescentes, acerca de su sexualidad, fundamentalmente en el período de la adolescencia propiamente dicho. Es frecuente encontrarse con adolescentes, varones y mujeres que, aunque poseen algunos conocimientos acerca de los factores que influyen en la sexualidad, no conocen en qué medida influyen en ella. Modificando su comportamiento y aptitud inadecuados ante la sexualidad de los adolescentes (4).

Plan Internacional Ecuador realizó un estudio en el año 2016, identificando los patrones culturales que influyen en la educación para la sexualidad de niñas, niños y adolescentes, en las provincias de Manabí, Santa Elena, Guayas y Bolívar, se concluyó que las familias de escasos recursos y los factores estructurales son determinantes de situaciones de hacinamiento y limitado acceso de las familias al ejercicio de sus derechos, lo cual crea situaciones favorables para la promiscuidad y de mayor riesgo para prácticas de incesto, abuso, violencia sexual y el embarazo prematuro no deseado (5).

Melo, en su estudio, concluyeron que los conocimientos sobre responsabilidad sexual que tienen los estudiantes son insuficientes para llevar a cabo una vida sexual responsable que implique la toma de decisiones y conductas seguras, a pesar de la gran cantidad de información existente sobre educación sexual aún esta no logra ser difundida adecuadamente para satisfacer las
Vol. 4, Nro. 1, Publicado 2021-06-30

necesidades/inquietudes de los jóvenes adolescentes (6).

Macías expresa que los factores sociodemográficos tienen prevalencia en las adolescentes embarazadas causando el mayor número de embarazos que se da entre los 15 y 19 años de edad, que están cursando la educación secundaria y son madres solteras, se identificó que los patrones culturales que están presentes en la vida de las adolescentes es que actualmente sigue siendo un tabú en la sociedad. Y el desconocimiento influye en la prevalencia de embarazo en la adolescencia demostrando que en sus hogares no existe una buena comunicación efectiva sobre la sexualidad (7).

Para Quintero, los “Tabúes de la sexualidad y su influencia en la conducta de los adolescentes se ve afectada por los medios de comunicación como la Tv y el internet, proporcionan información real sobre la sexualidad, lo que trae consigo una percepción distorsionada sobre lo importancia de la sexualidad y conductas inapropiadas, los tabús sexuales en un futuro traerán consecuencias que afectaran a los jóvenes como la promiscuidad, ITS, embarazos no deseados entre otros (8).

López, en su publicación sobre la "Sexualidad en la adolescencia“, expresó que en nuestra cultura se fusiona la familia, la escuela y el sistema sanitario con el supuesto de que los adolescentes no tienen prácticas sexuales, estos han cambiado hace muy poco por las actitudes liberales de los adolescentes, expresando que las personas que trabajan en salud tienen la obligación de educar a los padres sobre la sexualidad de sus hijos cuando acudan a consultas médicas (9).

López (10), define a los Patrones Culturales como un conjunto de formas aprendidas de interactuar, en el marco de construir contextos para la acción. Son normas que se establecen en una región, ciudad, o país de acuerdo con las costumbres de un grupo de personas y van cambiando los avances, modificaciones y precisamente a esas costumbres que se van volviendo comunes en algún sitio. Las formas de organizar acciones señaladas al hablar de patrones culturales se aprenden simplemente al vivirlas como prácticas recurrentes.

Según Mendoza Toscan, en su artículo " La Actividad sexual temprana y el embarazo en la adolescencia“ indica que la sexualidad temprana en la adolescencia, representa un problema de salud pública por las consecuencias que conllevan: como el embarazo adolescente, el aumento de las infecciones de transmisión sexual, la infección por HIV/SIDA (segunda causa de muerte en los adolescentes) y los problemas familiares, económicos y sociales que se generan (11).

La influencia de la educación sexual en el hogar, escuelas, colegios y servicios de salud, deben promover la abstinencia sexual, las prevenciones del embarazo y las infecciones de transmisión sexual, 
son una las estrategias más eficaces para enfrentar estas problemáticas (11).

Por otra parte Viteri, en su publicación habla de los Patrones de violencia en las niñas de Ecuador, demostrando que los patrones ancestrales siguen activos en los hogares y que son interiorizados desde edades tempranas y que son causantes de provocar daños permanentes en la vida de estas niñas (12).

Marzall en su libro de Historia de la Antropología social define a los patrones culturales como: "toda experiencia humana queda culturalmente mediatizada, pues cada individuo interpreta la experiencia a bases de sus principios recibidos en la propia socialización y todas sus valoraciones son relativas al patrón cultural del que surgen (13).

Romero, define a los patrones culturales son un conjunto de normas que rigen el comportamiento de un grupo organizado de personas, en función de sus tradiciones, costumbres, hábitos, creencias, ubicación geográfica y experiencias, para establecer unos modelos de conductas. La cultura favorece la afinidad entre los individuos que viven en una misma sociedad, quienes se sienten identificados entre sí en su entorno comunitario (14).

Los patrones culturales se han de establecer de acuerdo con cada persona, ya que por el hecho de que pertenezcas a una región no necesariamente se tendría que seguir las costumbres, sino también adoptar otras que aporten de manera positiva de otras regiones o incluso países, sin imponer ideas y sin romper con lo establecido que es "normal" para la gente (15).

En Ecuador las cifras del Instituto Nacional de Estadística y Censos (INEC) indican que en 2019 se han registrado 51.711 nacidos vivos de "mujeres adolescentes" de 10 a 19 años. En la última década, Ecuador ha tenido tres estrategias distintas para educar y prevenir el embarazo de niñas y adolescentes. Entre 2011 al 2014 funcionó la estrategia intersectorial de planificación familiar y prevención del embarazo en adolescentes (ENIPLA) que promovía la educación sexual integral y era dirigida de forma conjunta por los ministerios de salud, educación, inclusión económica-social y el desaparecido ministerio coordinador de desarrollo social (16). Sin embargo, parecería que no hubo efecto porque lo que ocurre actualmente permite ver que el problema ha incrementado.

Manabí es una provincia con mayor índice de embarazos en adolescentes, Portoviejo es el cantón con mayor número de casos seguido de Santa Ana, 24 de Mayo y Junín. Pues sólo en la capital manabita en el 2017 se atendieron 9.141 adolescentes embarazadas entre 10 y 19 años. De ellas 169 recibieron atención por primera vez. En el 2018 se atendieron 11.378 casos de menores embarazadas. Y en el 2019 hasta junio reportaron 1.807 partos (17)
La mayoría de los casos de embarazos en niñas y adolescentes sucedieron cuando se encontraban fuera del sistema educativo, su condición de embarazo las obligó a dejarlo, influyendo en las oportunidades educativas, laborales, seguridad económica. Como resultado de ello, las madres adolescentes, sus hijos, hijas y familias, tienen mayor vulnerabilidad a la pobreza y a la exclusión social.

La investigación es muy relevante nos permitirá identificar los patrones culturales que influyen en la sexualidad de las y los adolescentes de la comunidad de Chade, para intervenir educándolos correctamente y despejando sus inquietudes ante la importancia de saber llevar una vida sexual con responsabilidad que no represente daños en su integralidad como ser biopsicosocial.

Es necesario abordar la tasa mundial de embarazo adolescente para tener una visión de cómo se relacionan los patrones al problema de estudio. En ese sentido en estudios realizados se estima que ocurren 46 nacimientos por cada 1.000 niñas, mientras que las tasas de embarazo adolescente en América Latina y el Caribe continúan siendo las segundas más altas en el mundo (18). Esto hace visible el problema y entrar a indagar por qué ocurre estos hechos.

Considerando la problemática planteada se estableció el siguiente objetivo general: Determinar los patrones culturales que influyen en la sexualidad de los adolescentes de la comunidad de Chade del cantón Jipijapa. Y se complementó con los siguientes objetivos específicos 1.-Identificar las Características sociodemográficas de la población adolescentes de la comunidad de Chade 2.Establecer los principales patrones culturales que influyen en la sexualidad y los riesgos para los adolescentes de la comunidad de Chade 3.Identificar las actitudes y prácticas sexuales de los adolescentes de la comunidad de Chade.

\section{Materiales y métodos}

El tipo de investigación utilizada fue el descriptivo, analítico no experimental, acercándose al conocimiento que tienen sobre los patrones culturales y su influencia en la sexualidad de los adolescentes, mediante las variables patrones culturales (independiente) y la sexualidad (dependiente). Se aplicó el método deductivo y descriptivo, los instrumentos de la investigación fueron la encuesta aplicada a través de la visita domiciliaria, para la recolección de datos la misma que estuvo dirigida a las y los adolescentes de la comunidad de Chade zona rural del cantón Jipijapa. La encuesta estuvo estructurada por 44 preguntas divididas en tres 4 aspectos importantes como: sociodemográfico, conocimiento, de actitudes y prácticas sexuales.

La población objeto de estudio son los adolescentes de la comunidad de Chade que corresponde a $56 \mathrm{de}$ 
acuerdo con datos obtenidos en el ASIS de la comunidad realizado en el año 2019-2020.

La muestra seleccionada fueron 30 adolescentes, hombres y mujeres pertenecientes de la comunidad. Los criterios de inclusión considerados fue la población comprendida entre 10 y 19 años de ambos sexos que habitan en la comunidad de Chade, y los criterios de exclusión fueron los adolescentes que no quisieron participar en la investigación, hijos de madres que no dieron la autorización, (no firmaron el consentimiento), aquellos que se encontraban en clases en el momento de aplicar la encuesta, los que no se encontraban en la comunidad, y aquellos que no le dieron importancia al estudio.

\section{Resultados}

La comunidad de Chade tiene alrededor de 65 familias de etnia montubia, de habla española y clima tropical con un total de 56 adolescentes entre las edades de 10 a 19 años, de los cuales 30 participaron el proyecto. Predomina el género femenino, la edad que prevalece en las y los adolescentes varía entre los 12 y 14 años, la gran mayoría de los jóvenes son de religión católica y de estado civil soltero con un $96.67 \%$, el $3.33 \%$ corresponde solo a un adolescente quien mantiene una relación estable (tabla No 1).

Respecto a la fecundidad solo el $3.33 \%$ de las adolescentes ha tenido un embarazo a 17 años, en educación el $73.33 \%$ esta cursado el nivel básico. Los adolescentes en un $53.33 \%$ conocen que son los patrones culturales, el $40 \%$ no tiene definido este concepto, el $2 \%$ piensan que son los roles asignados por la sociedad, en referencia al género el $66.67 \%$ no define este concepto, el $20 \%$ tiene claro la definición, en referencia al abuso sexual el $80 \%$ tiene muy claro que un abuso sexual, pero el $16.66 \%$, piensa diferente.

Respecto a discriminación el $86.67 \%$, tienen claro el significado, manifestando el rechazada por su sexo religión, origen y orientación sexual, el $96.67 \%$ indicaron que el machismo es cuando un hombre golpea o maltrata a una mujer, el $60 \%$ señalo que la sexualidad son las características anatómicas fisiológicas emocionales afectivas y de conducta del ser humano, el $56.67 \%$ respecto a la orientación sexual indico que es recibir orientación mediante charla, llegando a la conclusión que desconocen esta terminología.

Sobre los derechos sexuales el $36.67 \%$ indicaron que es, no ser discriminado por género, orientación sexual, embarazo o vivir con VIH, y el $36.67 \%$ señalo que los derechos sexuales es tomar decisiones libres responsables sobre la salud $\mathrm{y}$ vida reproductiva. (Tabla No 3). En referencia al conocimiento sobre sexualidad el $63.33 \%$ indicaron que sus padres si les han orientado sobre la sexualidad y el 36.67 no han recibido educación por parte de los padres.
Vol. 4, Nro. 1, Publicado 2021-06-30

Hablar de sexualidad con los padres el 53.33\% considera que es bueno, el $16.67 \%$ que es interesante y prohibido hablar del tema en el hogar, el $76.67 \%$ prefieren hablar de sexualidad con sus padres, el $13.33 \%$ con familiares y $80 \%$ indico que los conocimientos adquiridos sobre sexualidad lo obtuvieron en los centros educativos y $3.33 \%$ en las redes sociales.

Sobre las causas de embarazos en adolescentes, el $50 \%$ indico que hay desconocimiento, el $20 \%$ por amor a la pareja y el $16.66 \%$ por no utilizar métodos anticonceptivos. Sobre el conocimiento de los métodos anticonceptivos el $43.33 \%$ tienen idea sobre el preservativo, el $26.66 \%$ conocen de los anticonceptivos inyectables porque sus madres lo utilizan. (tabla No 4)

El $90 \%$ indico que las infecciones de transmisión sexual se contagia por relaciones sexuales, el $10 \%$ por picadura de mosquito, el $50 \%$ refieren que el contagio de ITS es por tener relaciones sexuales con personas infectadas, siendo los jóvenes más propensos a padecer estas enfermedades por tener una vida sexual activa, el $90 \%$ opina que tener varias parejas sexuales aumenta el riesgo para contagiarse de las infecciones de transmisión sexual, el 73.33\% opino que las prácticas sexuales de mayor riesgo de contagio de ITS son el sexo vaginal, oral y anal (Tabla No 5).

El $93.33 \%$ concuerda que las y los adolescentes no deben ser obligados para iniciar su relación sexual, el $90 \%$ de los encuestados opinan que cuando un hombre maltrata o agrede a una mujer es más hombre respondiendo que no, un porcentaje de $83.33 \%$ considera inaceptable una relación de un adulto con una adolescente y un $16.67 \%$ lo considera aceptable siendo un factor predominante en nuestra sociedad, donde varias de la veces si la pareja tiene posibilidades económicas y bienes materiales terminan aceptando los padres esta relación, el 50\% de los adolescentes refiere que la edad adecuada para el inicio de la relaciones sexuales es a la edad de 18 años, sin embargo un $43.33 \%$ piensa que sería adecuado no tener relaciones sexuales hasta el casamiento de acuerdo a su criterio propio.

De los 30 adolescentes, 19 de ellos manifiestan que el método anticonceptivos más utilizado por los adolescentes es el preservativo con un $63.33 \%$, el $43.33 \%$ refiere que para prevenir un embarazo y evitar una ITS no tendría relaciones sexuales y un $30 \%$ primero se informaría del tema antes de actuar, el $86.67 \%$ se haría responsable si quedara embarazada o su pareja se embarace y un $3.33 \%$ buscaría ayuda de un amigo para hablar de este tema importante, de los 4 adolescentes que han tenía práctica sexual 2 de ellos si han utilizado métodos anticonceptivos equivalente a un $6.67 \%$ uno no utilizo y el otro no conoce los métodos anticonceptivos, los 26 restantes aun no experimentan las relaciones sexuales, de los 4 activos el $3.33 \%$ ha utilizado el método 
anticonceptivo natural el $6.67 \%$ utilizo preservativo $y$ un $3.33 \%$ que es una adolescente utiliza el implante subdérmico y los 26 restantes no responden.

Según los datos evidenciados el $86.67 \%$ de los adolescentes hombres y mujeres aún no inician sus relaciones sexuales, mientras que el $13.33 \%$ ya la han iniciado entre las edades de 16 a 18 años, 3 son de sexo femenino y 1 masculino, el $10 \%$ inicio su relación sexual con su enamorado y el 3,33\% inicio
Vol. 4, Nro. 1, Publicado 2021-06-30

con una trabajadora sexual por motivos de experimentar placer, el $86.67 \%$ aun no experimenta la práctica sexual pero un $13.33 \%$ realiza su práctica con la manipulación en zonas excitables de su cuerpo, de las 4 personas que ya han experimentado tener relaciones el $6,67 \% \mathrm{Si}$ ha tenido relaciones sexuales orales como anales y el $6,67 \%$ no responden.

Tabla 3. Conocimiento sobre los Patrones Culturales

\begin{tabular}{|c|c|c|c|c|}
\hline Orden & Alternativa & $\mathbf{F}$ & $\%$ & Total \\
\hline \multirow{3}{*}{$\begin{array}{l}1 \text { ¿Para } r \text { usted } \\
\text { patrones culturales } \\
\text { son? }\end{array}$} & $\begin{array}{l}\text { Relaciones familiares, valores, sentimientos } \\
\text { propios en las personas. }\end{array}$ & 12 & 40 & \multirow{3}{*}{100} \\
\hline & Son roles asignados por la sociedad. & 2 & 6.67 & \\
\hline & $\begin{array}{l}\text { Creencias, mitos, costumbres, tradiciones y } \\
\text { prácticas de la población. }\end{array}$ & 16 & 53.33 & \\
\hline \multirow{3}{*}{$\begin{array}{l}2 \text { ¿Para usted género } \\
\text { es? }\end{array}$} & Se refiere a dos sexo, hombre y mujer & 6 & 20 & \multirow{3}{*}{100} \\
\hline & $\begin{array}{l}\text { Construcción cultural y social relacionada con lo } \\
\text { masculino y lo femenino }\end{array}$ & 4 & 13.33 & \\
\hline & $\begin{array}{l}\text { Conjunto de roles, papeles y normas } \\
\text { determinados para hombres y mujeres }\end{array}$ & 20 & 66.67 & \\
\hline \multirow[t]{3}{*}{$\begin{array}{l}3 \text { ¿Para Ud. abuso } \\
\text { sexual es? }\end{array}$} & $\begin{array}{l}\text { Cuando un adulto engaña, seduce, o intimide a } \\
\text { un adolescente para tener contacto físico de } \\
\text { naturaleza sexual, con aparente consentimiento. }\end{array}$ & 5 & 16.67 & \multirow{3}{*}{100} \\
\hline & $\begin{array}{l}\text { Cuando un adulto toma por la fuerza aun } \\
\text { adolescente para cometer un acto sexual, incluye } \\
\text { comentarios, insinuaciones sexuales. }\end{array}$ & 24 & 80 & \\
\hline & $\begin{array}{l}\text { Todo acto que atente con la integridad de una } \\
\text { persona por identificarse por un género } \\
\text { determinado. }\end{array}$ & 1 & 3.33 & \\
\hline \multirow[t]{3}{*}{$\begin{array}{l}4 \text { ¿Para Usted la } \\
\text { discriminación es? }\end{array}$} & $\begin{array}{l}\text { Cuando una persona es rechazada por su sexo, } \\
\text { religión, origen étnico, edad, embarazo u } \\
\text { orientación sexual. }\end{array}$ & 26 & 86.67 & \multirow[t]{3}{*}{100} \\
\hline & $\begin{array}{l}\text { Cuando es respetada y valorada en un } \\
\text { determinado grupo. }\end{array}$ & 2 & 6.67 & \\
\hline & Ninguna de los anteriores. & 2 & 6.67 & \\
\hline \multirow{4}{*}{$\begin{array}{l}5 \text { ¿Para Usted el } \\
\text { machismo es? }\end{array}$} & Superioridad de la mujer & 0 & 0 & \multirow{4}{*}{100} \\
\hline & Cuando un hombre maltrata o golpea a una mujer & 29 & 96.67 & \\
\hline & Inferioridad del género masculino & 1 & 3.33 & \\
\hline & $\begin{array}{l}\text { Que el hombre es por naturaleza superior a la } \\
\text { mujer }\end{array}$ & 0 & 0 & \\
\hline \multirow{3}{*}{$\begin{array}{l}6 \text { ¿Para Usted la } \\
\text { sexualidad es? }\end{array}$} & Tener relaciones sexuales. & 8 & 26.67 & \multirow{3}{*}{100} \\
\hline & $\begin{array}{l}\text { Son características anatómicas, fisiológicas, } \\
\text { emocionales, afectivas y de conducta del ser } \\
\text { humano. }\end{array}$ & 18 & 60 & \\
\hline & Relación afectiva que existe entre las personas. & 4 & 13.33 & \\
\hline \multirow{2}{*}{$\begin{array}{l}7 \quad \text { ¿Para Usted } \\
\text { Orientación sexual es? }\end{array}$} & Diferencia que existe hombre y mujeres & 9 & 30 & \multirow[b]{2}{*}{100} \\
\hline & $\begin{array}{l}\text { Recibir orientación en sexualidad mediante } \\
\text { charlas }\end{array}$ & 17 & 56.67 & \\
\hline
\end{tabular}


Preferencia o inclinación sexual hacia personas $4 \quad 13.33$

de su mismo sexo o del sexo opuesto

8 de los siguientes No ser discriminado por géneros, orientación $11 \quad 36.67$

¿Cuál considera Usted sexual, embarazo, por vivir con VIH

que son derechos A decidir cuándo y cuantos hijos/as tener. $\quad 1 \quad 3.33 \quad 100$

$\begin{array}{llll}\text { sexuales: } & \text { Tomar decisiones libres, informadas, voluntarias } & 7 & 23.33\end{array}$

y responsables, sobre su sexualidad, y su vida y

su orientación sexual.

A tomar decisiones libres, responsables e $11 \quad 36.67$

informadas sobre su salud y su vida reproductiva.

Fuente: Adolescentes de la comunidad de Chade

Tabla 4. Conocimiento sobre la sexualidad

\begin{tabular}{|c|c|c|c|c|}
\hline Orden & Alternativa & $\mathbf{F}$ & $\%$ & Total \\
\hline \multirow{2}{*}{$\begin{array}{l}\text { 9. ¿Sus padres le han instruido sobre } \\
\text { sexualidad? }\end{array}$} & $\mathrm{Si}$ & 19 & 63.33 & \multirow[b]{2}{*}{100} \\
\hline & No & 11 & 36.67 & \\
\hline \multirow{4}{*}{$\begin{array}{l}\text { 10. Hablar sobre sexualidad con sus } \\
\text { padres es un tema considerado como: }\end{array}$} & Interesante & 5 & 16.67 & \multirow{4}{*}{100} \\
\hline & Bueno & 16 & 53.33 & \\
\hline & Malo & 4 & 13.33 & \\
\hline & Prohibido & 5 & 16.67 & \\
\hline \multirow{5}{*}{$\begin{array}{l}\text { 11. ¿Con quién prefiere Usted hablar } \\
\text { sobre sexualidad? }\end{array}$} & Padres & 23 & 76.67 & \multirow{5}{*}{100} \\
\hline & Amigos & 1 & 3.33 & \\
\hline & Familiares & 4 & 13.33 & \\
\hline & Pareja & 2 & 6.67 & \\
\hline & Maestro/a & 0 & - & \\
\hline \multirow{4}{*}{$\begin{array}{l}\text { 12. Los conocimientos adquiridos } \\
\text { sobre sexualidad donde los obtuvo: }\end{array}$} & $\begin{array}{l}\text { Escuela/ } \\
\text { Colegio }\end{array}$ & 24 & 80 & \multirow{4}{*}{100} \\
\hline & Familia & 5 & 16.67 & \\
\hline & $\begin{array}{l}\text { Redes } \\
\text { sociales }\end{array}$ & 1 & 3.33 & \\
\hline & Con amigos & 0 & 0 & \\
\hline \multirow{6}{*}{$\begin{array}{l}\text { 13. Para usted cuál es la causa } \\
\text { principal de un embarazo en las } \\
\text { adolescentes }\end{array}$} & $\begin{array}{l}\text { Desconocimiento } \\
\text { sobre la sexualidad }\end{array}$ & 15 & 50 & \multirow{6}{*}{100} \\
\hline & Por Experimentar & 2 & 6.67 & \\
\hline & $\begin{array}{l}\text { Por no utilizar } \\
\text { métodos } \\
\text { anticonceptivos }\end{array}$ & 5 & 16.67 & \\
\hline & Por Amor & 6 & 20.00 & \\
\hline & Por Obligación & 0 & 0 & \\
\hline & Abuso sexual & 2 & 6.67 & \\
\hline \multirow[t]{8}{*}{$\begin{array}{l}14 \text { ¿Qué métodos anticonceptivos } \\
\text { conoce? }\end{array}$} & $\begin{array}{l}\text { Naturales: } \\
\text { (eyacular afuera, } \\
\text { ritmo, } \\
\text { temperatura, moco } \\
\text { cervical) }\end{array}$ & 3 & 10 & \multirow[t]{8}{*}{100} \\
\hline & Lactancia materna & 0 & 0 & \\
\hline & Preservativos & 13 & 43.33 & \\
\hline & $\begin{array}{l}\text { Anticonceptivos } \\
\text { orales }\end{array}$ & 3 & 10 & \\
\hline & $\begin{array}{l}\text { Píldora de } \\
\text { emergencia }\end{array}$ & 0 & 0 & \\
\hline & DIU (T de cobre) & 0 & 0 & \\
\hline & $\begin{array}{l}\text { Anticonceptivos } \\
\text { inyectables }\end{array}$ & 8 & 26.67 & \\
\hline & $\begin{array}{l}\text { Implantes } \\
\text { Subdérmicos }\end{array}$ & 3 & 10 & \\
\hline
\end{tabular}


Fuente: Adolescentes de la comunidad de Chade

Tabla 5. Conocimiento sobre las infecciones de transmisión sexual

\begin{tabular}{|c|c|c|c|c|}
\hline Orden & Alternativa & $\mathbf{F}$ & $\%$ & Total \\
\hline \multirow{4}{*}{$\begin{array}{l}15 \quad \text { ¿Una infección } \\
\text { transmisión sexual es? }\end{array}$} & $\begin{array}{l}\text { Es una infección que se produce por falta } \\
\text { de aseo de genitales }\end{array}$ & 0 & 0 & \multirow{4}{*}{100} \\
\hline & $\begin{array}{l}\text { Es una infección que se puede transmitir } \\
\text { por picadura de insectos }\end{array}$ & 2 & 6.67 & \\
\hline & $\begin{array}{l}\text { Es una infección que se produce solo por } \\
\text { contacto homosexual }\end{array}$ & 1 & 3.33 & \\
\hline & $\begin{array}{l}\text { Es una infección que se puede transmitir } \\
\text { por contacto sexual }\end{array}$ & 27 & 90 & \\
\hline \multirow{5}{*}{$\begin{array}{l}16 \text { ¿Sabe cómo una persona se } \\
\text { contagia de las infecciones de } \\
\text { transmisión sexual (ITS)? }\end{array}$} & Por un beso & 4 & 13.33 & \multirow{5}{*}{100} \\
\hline & Saludar o abrazar a una persona infectada & 3 & 10 & \\
\hline & $\begin{array}{l}\text { Compartir el mismo servicio higiénico } \\
\text { (baño) }\end{array}$ & 3 & 10 & \\
\hline & $\begin{array}{l}\text { Relaciones sexuales con una persona } \\
\text { infectada }\end{array}$ & 15 & 50 & \\
\hline & De madre a hijos & 5 & 16.67 & \\
\hline \multirow{3}{*}{$\begin{array}{l}17 \text { ¿El tener varias parejas } \\
\text { sexuales es un riesgo para } \\
\text { contagiarse de las infecciones de } \\
\text { transmisión sexual (ITS)? }\end{array}$} & $\mathrm{Si}$ & 27 & 90 & \multirow[b]{3}{*}{100} \\
\hline & No & 0 & 0 & \\
\hline & No sé & 3 & 10 & \\
\hline \multirow{4}{*}{$\begin{array}{l}18 \text { ¿Cuál de las siguientes } \\
\text { prácticas sexuales es de mayor } \\
\text { riesgo para el contagio de } \\
\text { infecciones de transmisión } \\
\text { sexual? }\end{array}$} & Sexo vaginal & 6 & 20.00 & \multirow{4}{*}{100} \\
\hline & Sexo oral (boca) & 2 & 6.67 & \\
\hline & Sexo anal & 0 & 0 & \\
\hline & Todas & 22 & 73.33 & \\
\hline
\end{tabular}

Fuente: Adolescentes de la comunidad de Chade

\section{Discusión}

La adolescencia es reconocida como una etapa prioritaria, especialmente en los ámbitos de salud sexual y reproductiva, al acceso apropiado a los servicios de salud que ofrece nuestro país respetando los derechos humanos.

La población de estudios varia en las edades de 10 a 19 años, el $73.33 \%$ la mayoría está cursando el nivel secundario, siendo solteros con un $96.67 \%$ de los adolescentes y un $3.33 \%$ unión libre, se pudo identificar que el $53.33 \%$ tienen conocimientos sobre los patrones culturales y el $60 \%$ conoce sobre la sexualidad, en comparación a un estudio realizado en la provincia de Manabí en la comunidad de Buenos Aires donde el $80 \%$ de los adolescentes encuestados creían que la sexualidad es solo tener relaciones sexuales evidenciándose que sus conocimientos eran bajos en estos temas.

En la tabla $\mathrm{N}^{\circ} 8$ el $86.67 \%$ de los encuestados no han experimentado tener relaciones sexuales aun, sin embargo, en un estudio realizado en Cuba detallan que las conductas sexuales a inicio temprano son consideradas como un riesgo señalando que los adolescentes de 13 años realizan estos actos con su pareja por amor, placer o diversión sin protección alguna (19).

La educación sexual cuyos padres influyen en ellos es de un $63.33 \%$, si bien estudios anteriores expresan que la influencia familiar es fundamental en la determinación del comportamiento sexual de sus hijos, a través de la comunicación transmitida en el hogar por los valores, acciones y comportamientos sobre sexualidad (20).

Fernández expresa que la comunicación sobre sexualidad entre padres/madres y adolescentes enfrenta dificultades particulares producto de factores socioculturales por la conducta que tienen los adolescentes latinos y el temor que tienen al hablar estos temas en sus hogares (21).

A través de las visitas domiciliarias en la comunidad de Chade y durante la aplicación del instrumento (encuesta), se constató que existe un porcentaje significativo que no tiene claro que son los patrones culturales ni mucho menos como interfieren en la sexualidad, tema que para algunos jóvenes que se encuentran en el proceso de la pubertad ente los 10 a 12 años, desconocían ciertos términos relacionados con la educación sexual.

\section{Conclusiones}

$>$ La comunidad de Chade es zona rural, prevaleciendo el sexo femenino, y entre las edades de 12 a 14 años, predomina la religión católica, estado civil soltero y la mayoría está cursando la educación básica, y viven con sus padres.

$>$ Los principales riesgos de los adolescentes de la comunidad de Chade, es el bajo conocimiento, insuficiente participación de los padres en la educación sexual de sus hijos, piensan que aún son muy pequeños para hablar de estos temas, 
debido a su nivel cultural y tabúes que persisten en la comunidad.

$>$ Los adolescentes expresaron que no deben ser obligados a iniciar su actividad sexual, que es voluntad propia y que la mayoría lo desearía experimentar a los 18 años cuando sus conocimientos estén claros y no exista el temor o riesgo de contraer un embarazo no deseado o una enfermedad de transmisión sexual que ponga en riesgo su salud sexual, reproductiva sin afectar su estado psicosocial.
Vol. 4, Nro. 1, Publicado 2021-06-30

$>$ Ante los resultados obtenidos sobre el leve déficit de conocimiento se plantea una propuesta educativa dirigida a los adolescentes de la comunidad de Chade para mejorar su situación de salud en especial la sexualidad previniendo complicaciones, como embarazos no deseados, enfermedades de trasmisión sexual, aborto, adicciones y complicaciones en su estilo de vida.

\section{Bibliografía}

1. Yeral EC. scielo. [Online]. Quito-Ecuador: Ediciones Abya-Yala; 2017 [cited 2021 Enero 5. Available from: http://scielo.sld.cu/pdf/hmc/v17n3/hmc10317.pdf.

2. Cervantes L. repository. [Online].; 2018 [cited 2021 Febrero 15. Available from: https://repository.ucc.edu.co/bitstream/20.500.12494/5268/1/FACTORES\%20QUE\% 20INFLUYEN\%20EL\%20DESARROLLO\%20DE\%20LA\%20SEXUALIDAD\%20DE\%20LOS\% 20ADOLESCENTES.pdf.

3. Callo KZC. repositorios. [Online].; 2018 [cited 2021 Febrero 15. Available from: http://repositorio.unap.edu.pe/bitstream/handle/UNAP/7720/Calle_Callo_Karin_Zuberbi.pdf ?sequence $=1 \&$ is Allowed $=\mathrm{y}$.

4. London Y. Conocimiento en adolescentes de Factores que Influyen en la Expresion de la Sexualidad. RevInf Cient. 2017 Marzo;I(1028-9933).

5. Ecuador PId. Resultados de estudios de patrones culturales en la educacion para la sexualida de niás. niños y adolescentes. CSSR ECUADOR. 2016 Noviembre; II.

6. Alejandra MTM. repositorio. [Online].; 2018 [cited 2021 Febrero 15. Available from: http://repositorio.uta.edu.ec/bitstream/123456789/27881/2/Tesis\%20completa\%20PDF.pdf.

7. Moreira AKM. repositorio. [Online].; 2019 [cited 2021 Febrero 16. Available from: http://repositorio.unesum.edu.ec/bitstream/53000/1860/1/UNESUM-ECUADOR-ENFERMERIA-2019-70.pdf.

8. Joyner QMI. reposotorio. [Online].; 2019 [cited 2021 Febrero 16. Available from: http://repositorio.unemi.edu.ec/bitstream/123456789/4804/1/2.\%20TABUES\%20DE\%20LA\% 20SEXUALIDAD\%20Y\%20SU\%20INFLUENCIA\%20EN\%20 LA\%20CONDUCTA\%20DE\%20LOS\%20ADOLESCENTES\%20DE\%208VO\%20A\%C3\% 910\%20DE\%20BASIC.pdf.

9. Sanchez FL. Sexualidad en la Adolescencia. Regreso a las Bases. 2017; XXI(278).

10. Mero JL. Los patrones culturales que predominan en la familia. Polo de Conocimiento. 2017 Septimbre; 2(9).

11. Toscan LAM. La Actividad Sexual Temprana y el Embarazo en la Adolescencia. Revista Chilena de Obstetricia. 2016 Junio; 81(3).

12. Maria Amelia Viteri P. Patrones de Violencia en la niñas de Ecuador. Revista Bitacora Academica. 2017 Junio; I(3)

13. Marzal M. dspce. [Online]. Quito, Ecuador: Ediciones Abaya Yala; 2016 [cited 2021 Enero 5.

14. Romero ML. Patrones Culturales. Lifeder. 201914 de Noviembre; I(5).

15. Rosales J. scribd. [Online]. [cited 2021 Febrero 16. Available from: https://es.scribd.com/document/111600926/Los-Patrones-Culturales.

16. Torre VGdl. Salud Publica Embarazo en adolescentes durante la Pandemia. Edicion Medica. 2019 Marzo; II.

17. Manabi C. Ceibonews. [Online].; 2019 [cited 2021 Febrero 8.

18. Chile O. America Latina y el Caribe tienen la segunda tasa alta de embarazos de adolescentes en el mundo. Paho. 2018 Febrero; III(996).

19. Figueroa LA. Abordaje teórico en el estudio de las conductas sexuales de riesgo en la adolescencia. Rev Ciencias Médicas Scielo. 2019 Noviembre; 23(VI).

20. Cabrera-García V. Scielo. [Online].; 2018 [cited 2021 Abril 8. Available from: 
https://www.scielosp.org/article/rsap/2018.v20n3/279-285/.

21. Ana Michelle Fernández. Us National Library of Medicine. [Online].;

Julio 21 del 2017 [cited 2021 Marzo 21. Available from:https://www.ncbi.nlm.nih.gov/pmc/articles/PMC5520656/. 